\title{
Wie viel Schlaf brauchen Chirurgen?
}

Chirurgische Eingriffe, die von Ärzten nach dem Nachtdienst durchgeführt werden, gehen insgesamt betrachtet nicht mit einer signifikant erhöhten Komplikationsrate einher. Allerdings steigt diese an, sobald die Schlafdauer während des Dienstes sechs Stunden unterschreitet.

- Wie es um die Leistungsfähigkeit von operierenden Ärzten nach einem Nachtdienst bestellt ist, wurde in einer retrospektiven Kohortenstudie untersucht. Dabei verglich man die Komplikationen von 919 chirurgischen und 957 gynäkologischen operativen Eingriffen bei einer Gruppe von $86 \mathrm{Chi}$ rurgen und 134 Gynäkologen, welche diese in den Jahren 1999 bis 2008 nach dem Nachtdienst durchgeführt hatten, mit 3552 bzw. 3945 Kontrolleingriffen, die jeweils nach einer Nacht ohne Dienst von denselben Personen durchgeführt wurden.
Die Komplikationsrate der Eingriffe lag mit 101 in der Postnachtdienstperiode und 365 bei den Kontrolleingriffen annähernd gleich hoch $(5,4$ vs. 4,9\%). Eine genauere Analyse ergab jedoch ein etwas anderes Bild. In der Gruppe von Ärzten, die sechs Stunden oder weniger Gelegenheit zum Schlaf hatten, kam es bei 82 von 1317 Eingriffen zu Komplikationen, bei einer Gelegenheit zum Schlaf von über sechs Stunden dagegen nur bei 19 von 559 Eingriffen. Dies ergab einen signifikanten Unterschied von 6,2 vs. $3,4 \%$.
KOMMENTAR: Aufgrund der komplexen und nicht leicht valide zu erhebenden Datenlage ist es nicht schwer, die Untersu- chung methodisch anzugreifen. Allerdings erscheint es ohnehin fraglich, ob man einen derart evidenten Zusammenhang zwischen Übermüdung und Fehlleis- tungen, der auch im Alltag bei weit gerin- gerer psychophysischer Belastung jedem

bekannt ist, unbedingt hieb- und stichfest durch eine wissenschaftliche Studie untermauern muss. Jeder, der selbst einmal Nachtdienst in der Klinik gemacht hat, weiß, dass bei einer Schlafdauer von weniger als vier Stunden, aber auch bei häufigen Unterbrechungen eines theoretisch längeren Schlafes man an sich selbst zumindest eine gewisse Unkonzentriertheit feststellt. Die individuellen Unterschiede dabei sind enorm gro $\beta$, und zum Glück führen kleinere Fehler nicht immer zur großen Katastrophe.

H. S. FÜEßL =

\section{- J. M. Rothschild et al. \\ Risks of complications by attending physi- cians after performing nighttime procedures. JAMA 302 (2009) 14, 1565-1572}

\section{Wo ist die Milz geblieben?}

— Eine gesunde 23-jährige kinderlose Frau ohne Vorerkrankungen stellte sich wegen Unterbauchschmerzen vor. Im mittleren Unterbauch fand sich eine tastbare Raumforderung. Bei der Abdomensonografie lag die Milz nicht an der normalen Stelle. Die tastbare Raumforderung konnte einer homogenen echoarmen Weichteilstruktur von 11 x 9 x 4 cm Größe im Bereich des Beckens zugeordnet werden. Man führte eine Leber- und Milzszintigrafie durch (Abb. links), die neben der Speicherung in der Leber eine Speicherung im Bereich des unteren Abdomens ergab. Die Multislice-CT mit dreidimensionaler Rekonstruktion (Abb. rechts) ließ eine Milz im Bereich des Beckens erkennen

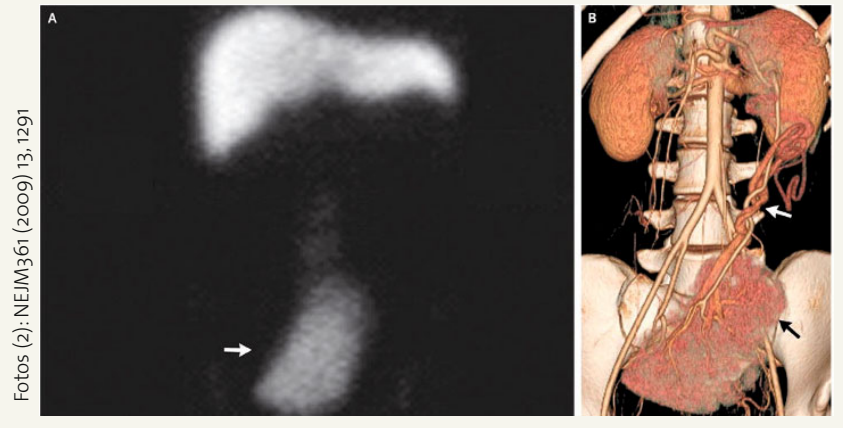

Technetium-Szintigrafie (links) und dreidimensionale Rekonstruktion des Multislice-CT (rechts).

(schwarzer Pfeil), die an einem langen Gefäßstiel hing (weißer Pfeil). Eine Wandermilz oder Beckenmilz entsteht durch eine ungewöhnliche Dehnbarkeit oder Malformation der Milzhaltebänder. Potenzielle Komplikationen sind eine Torsion des langen Gefäßstils, und ein nachfolgender Milzinfarkt. Die Behandlungs möglichkeiten sind eine Splenopexie oder eine Splenektomie. Die Patientin sprach sich gegen eine Operation aus. Diese Entscheidung liegt drei Jahre zurück, und der Patientin geht es bis heute gut.

H. S. FüEßL =

- C.-A. Tseng, A.-L. Chou

(Buddhist Dalin Tzu Chi General Hospital Chia-Yi, Taiwan): Pelvic spleen. New Engl. J. Med. 361 (2009) 13, 1291 